\title{
The Virtual Surgery of Extraocular Muscles Based on Gesture Interaction
}

\author{
Mingxuan Chen, Guanglong Du*, Ping Zhang and Chuangye Zhao \\ School of Computer Science and Engineering, South China University of Technology, Guangzhou 510006, China \\ ${ }^{*}$ Corresponding author
}

\begin{abstract}
Virtual surgery is a process which uses virtual reality technology to realize surgery simulation, it can provide doctors with real-life experience of visual, auditory, tactile and so on, and it also plays an important role in surgical training and planning. During the interactive process, we used Leap Motion Controller to obtain hand information such as user hand joint positions, posture, fingers. We used the feature vector extracted from the doctor's hand to recognize the gesture, and then completed the process of virtual surgery through a combination of gestures. In the end, this thesis verified the gesture control and filtering effect on the virtual surgery platform of extraocular muscles based on gesture interaction in the laboratory. The traditional virtual surgery system needs the doctor to perform the operation using mouse and keyboard and other touched equipment, which may pollute the environment of surgery. Our method uses hand gestures to perform the operation. It is not only natural and easy for doctor to control the machine, but also make the environment cleaner.
\end{abstract}

Keywords-gesture interaction; virtual surgery; extraocular muscle

\section{INTRODUCTION}

Computer network, software project management and image processing technology have played an important role in the continuous development of medicine since the computer application technology infiltrated the medical field gradually. It is a popular research through the use of the computer virtual reality technology [1] to help doctors to perform a surgery. Virtual surgery technology [2] is an important application of virtual reality technology in the medical field and it is a research area which fuses modern medicine, computer graphics, sensor technology and biomechanics. Virtual surgery generates the model of human's tissues and organs and the virtual operation environment then making simulation which can integrate with haptic device.

The research that applied the virtual reality technique into the simulation of surgery and the medical training has already developed for about thirty years. Reference [3] developed a 3D simulation system in simulating the transplant of the calf tendon in the 1980s. Dr. J. Levy built the virtual surgery system of hysteroscopy in 1995. And many universities and institutions carried out a deep research in the virtual surgery. Stanford University and Huston medical center have proven virtual surgery instrument technique. A laboratory in London brings the virtual surgery to the mobile client [4]. They developed software called Touch Surgery. The Doctor Thomas Gregory [5] in Descartes University used two GoPro vidicons to record the process of the virtual surgery. And the students can look back the entire process.

Human-computer interaction [6] is a key link in virtual surgery simulation. During the interaction process, we use Leap Motion Controller [7][8][9] to obtain hand information such as user hand joint positions, posture, fingers. We use the feature vector extracted from the doctor's hand to recognize the gesture, and then complete the process of Virtual Surgery through a combination of gestures. In order to ensure the accuracy and stability of the virtual devices in three dimensional spaces, we use Kalman Filter and Unscented Kalman Filter [10] to process user's original data in the position and attitude.

The remainder of this paper is organized as follows. Section II gives an overview of the extraocular muscles virtual surgery system. Section III describes the structure of the extraocular muscles virtual surgery system. Section IV details a serial of experiment. In Section V turns to the discussions and Section VI makes a conclusion.

\section{OVERVIEW}

The process of virtual surgery system based on gesture interaction is divided into the following steps: (1) Collecting the position and posture data of the operator's hand by Leap Motion sensor. (2) Doing a mapping between operator's gesture and concrete surgical operation according to the gesture recognition. (3) Implementing the related process of virtual surgery according to the intention of the operator.

The main research purpose of this article is using data collected from Leap Motion sensor to control operation equipment in the virtual scene [12]. In the process of virtual surgery, the movement of operator's hand is small, so the gesture data acquisition module adopts one sensor to acquire finger joints, palm position and other relevant information.

Gesture data preprocessing module mainly recognizes the gesture data and turns the operator's intentions into specific control information [13] [14]. Besides, the module need filtrate the gesture data since there was too much redundant data from Leap Motion sensor.

In virtual surgery system based on gesture interaction, the purpose of gesture interaction is to control the related operations of virtual surgery, including the location and the posture of specific surgical instruments in virtual surgery scenario. In virtual surgery, the accuracy of surgical 
instruments' pose is critical while the gesture data of operator's hand from Leap Motion sensor would be affected by environmental noise.

\section{VIRTUAL SURGERY SyStem BASEd ON THE GESTURE INTERACTION}

\section{A. Virtual Surgery System}

This paper uses extraocular muscle virtual surgery system based on gesture interaction to authenticate the key technology of virtual surgery system. This system, which is a control management procedure based on Microsoft Foundation Class, is composed of data acquisition module, gesture recognition module, data processing module and virtual surgery controlling module.

Date acquisition module mainly using Leap Motion sensor to obtain the information of operator's hand, such as characteristic description, position and posture. Before it works, it needs to be set the effective working range of the transducer. Gesture recognition module is mainly to identify the collected data. This paper defines eight common gestures which five types would be used to carry out interactive control of virtual surgery. Data processing module is mainly to deal with the operator's gesture data. According to the different of operator's hand and gestures we determine different operation, which is divided into the scene control and surgical equipment control two parts. In this module, gesture data for controlling equipment need be filter. Kalman Filter is used to optimize the location information of operator's hand while Unscented Kalman Filter optimizing the posture of operator's hand [10]. According to the different gestures, virtual surgery controlling module is used to control relevant scene and surgical equipment.

\section{B. The Establishment of Virtual Eyeball Model}

The object of virtual surgery based on gesture interaction presented this paper is extraocular muscle of human which is attached to the eyeball external muscle and responsible for eye movement. Normal every eye has six extraocular muscles which can be divided into rectus and oblique according to the direction of the muscle. There are four recti such as superior rectus, inferior rectus, medical rectus and lateral recuts. Clinically for record convenience, people often use the extraocular muscles in adhered on the surface of the eye location and the relative position on the edge of the cornea to locate the extraocular muscle. In normal, the four extraocular muscles locate behind cornea edge about $5.5 \mathrm{~mm}, 6.5 \mathrm{~mm}, 6.9$ $\mathrm{mm}$ and $7.7 \mathrm{~mm}$ respectively.

This paper experimental study aims at strabismus caused by extraocular muscle through the virtual surgery. So we simplified the eye tissue to eyeball, iris and cornea three parts and the eyeball were seen as a sphere. Extraocular muscles are simplified to four rectus evenly distributed in the first half eyeball and the part attached on surface of eyeball is simplified to ruled surface.

\section{The Mapping between Hand Gesture and Virtual Surgical Operation}

Leap Motion sensor can get six key points of operator's hand gesture, such as the position of palm's center and the position of five fingertips [15]. In addition, Leap Motion also provides us with the normal vector of palm and the information of hand's bending state. The six key points disperse in three-dimensional space, which is beneficial to gesture recognition. These key points can provide us with valid information as follows: (1) location information of key points which can describe the operator hand's coordinates in Leap Motion workspace. (2) Five vectors from palm's center to fingertips which can describe the finger pointing.

The gestures recognition [11] based on two-fork deciding tree is classified four classes, as shown in Figure I.

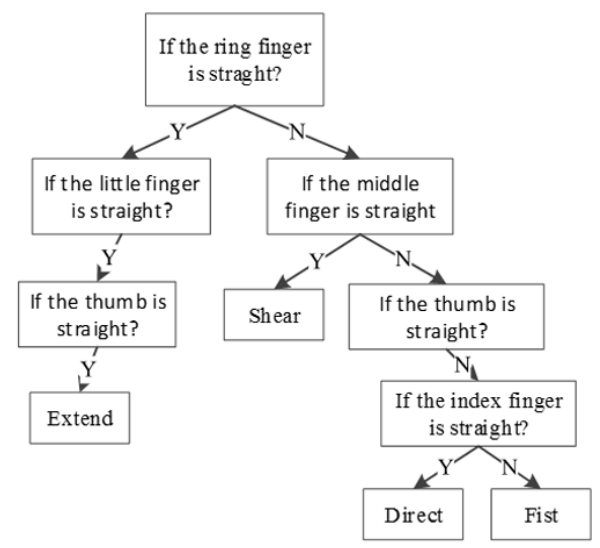

FIGURE I. THE GESTURES RECOGNITION TREE

The data collected from Leap Motion are on its own local coordinate system while controlling equipment in virtual surgery is on the world coordinate system. Meanwhile, the displacement of gesture is not the same as the actual moving distance of the equipment in virtual surgery. Therefore, the data need to be global coordinate conversion and set up a corresponding control proportion coefficient.

\section{EXPERIMENT}

\section{A. Contexts of Experiment}

To validate the presented virtual surgery, some related experiments have been carried out: (1) Operator use right hand to control the rotation of the eyeball which rotation parameters will be updated in real time according to the operator's hand gesture. After observing the state of the extraocular muscles when eye movement, operator makes the eye tissues return to the initial state. (2) Operator should adjust the scene camera through related gestures of left hand, and face the eye tissues at a comfortable angle of view. Then choose the extraocular muscle which needs to cut. (3) Operator use right hand to control the scalpel and move near extraocular muscle. When extraocular muscle contacts with the scalpel and detects a collision, the main view window shows the level of intersection between the extraocular muscle and the scalpel. What's more, it will produce an incision effect when the level 
reaches a threshold level. (4) In the same way as (3) but use right hand to control surgical scissor to cut the extraocular muscle.

The gestures of operator's hand and its specific function are shown in Table I.

TABLE I. THE SPECIFIC CONTROL MODE OF EXTRAOCULAR MUSCLE VIRTUAL SURGERY BASED ON GESTURE INTERACTION

\begin{tabular}{|c|c|c|}
\hline \multirow{2}{*}{ Gestures } & \multicolumn{2}{|c|}{ Hands } \\
\hline & Left hand & Right hand \\
\hline Fist & Null & $\begin{array}{l}\text { Recovery of eyeball and } \\
\text { extraocular muscle }\end{array}$ \\
\hline Extend & $\begin{array}{c}\text { Control distance of scene } \\
\text { camera }\end{array}$ & Control rotation of eyeball \\
\hline Direct & $\begin{array}{l}\text { Control rotation of scene } \\
\text { cameras }\end{array}$ & $\begin{array}{l}\text { Control pose and posture } \\
\text { of scalpel }\end{array}$ \\
\hline Shear & Scene camera recovery & $\begin{array}{l}\text { Control pose and posture } \\
\text { of scissor }\end{array}$ \\
\hline
\end{tabular}

\section{B. Results of Experiment}

The initial state of the whole system and the main interface of extraocular muscle virtual surgery are shown in Figure 2.

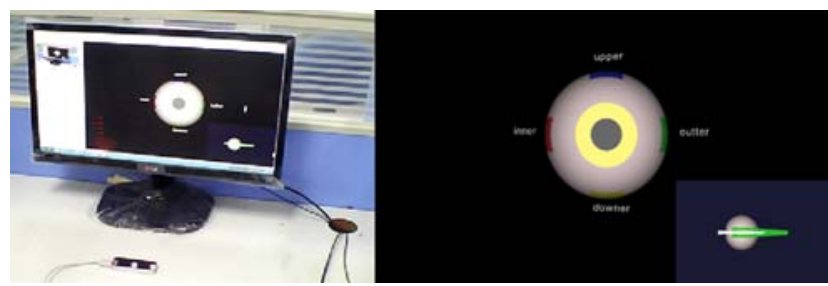

FIGURE II. THE ENVIRONMENT OF EXPERIMENT AND MAIN INTERFACE

The operator use left hand gesture Extend to move the camera closer, as shown in Figure 3.

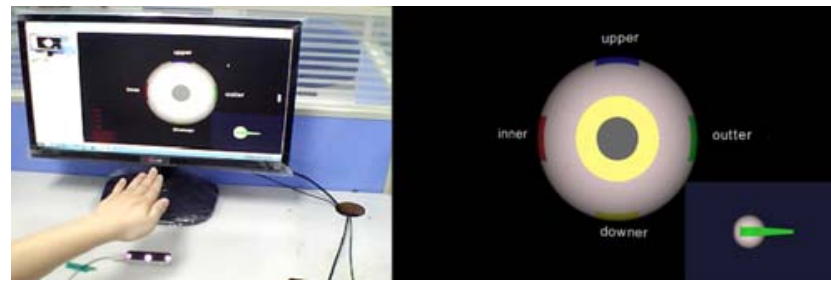

FIGURE III. THE LEFT HAND GESTURE EXTEND

The operator use right hand gesture Extend to rotate the eyeball. Figure 4 and Figure 5 are shown the eyeball look up and down respectively.

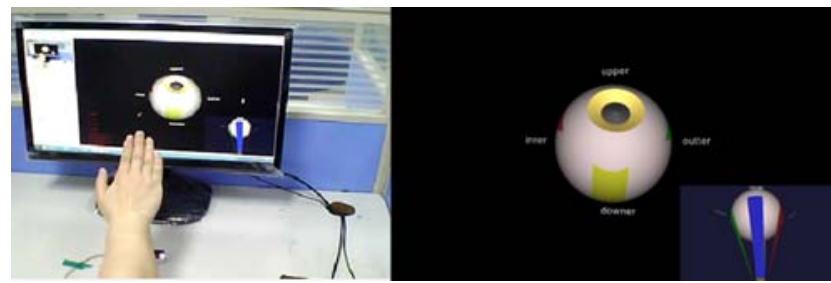

FIGURE IV. THE RIGHT HAND GESTURE EXTEND UP

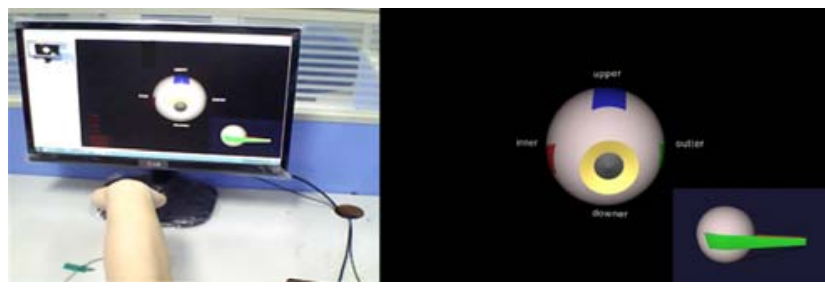

FIGURE V. THE RIGHT HAND GESTURE EXTEND DOWN

The operator use left hand gesture Direct to rotate the camera and face the extraocular muscle (lateral rectus, in green color), as shown in Figure 6.
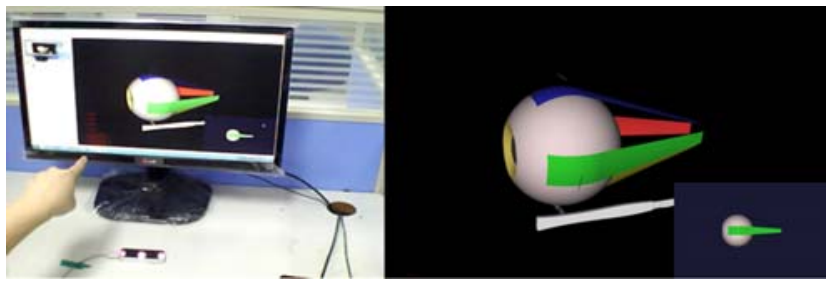

FIGURE VI. THE LEFT HAND GESTURE DIRECT

The operator uses right hand gesture Direct to control the virtual surgical scalpel and simulate the incision operation. Figure 7 shows the scalpel contacting with lateral rectus. Figure 8 shows the simulation of extraocular muscle fracturing and eyeball rotation.

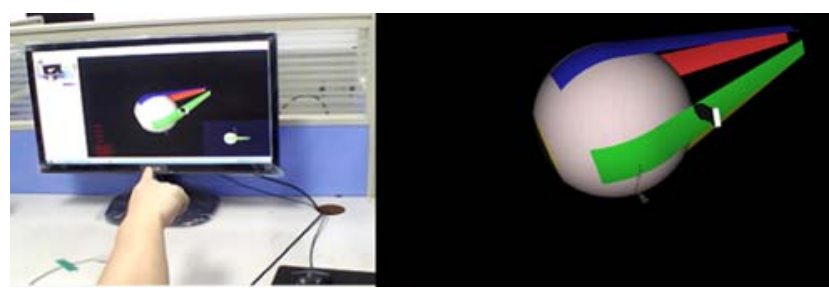

FIGURE VII. THE RIGHT HAND GESTURE DIRECT

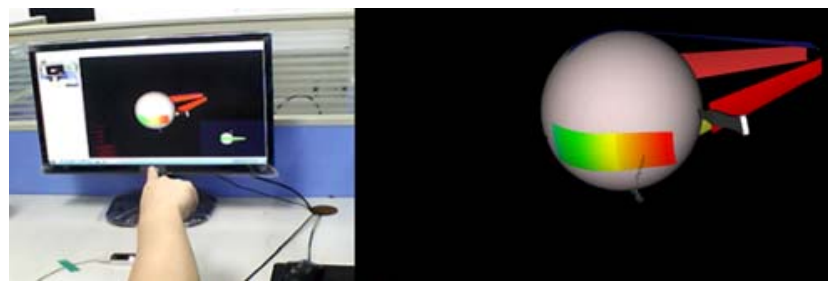

FIGURE VIII. THE SIMULATION OF INCISION OPERATION

The operator uses right hand gesture Shear to control the virtual surgical scissor and simulate the cutting operation. Figure 9 shows the scissor expanding as same as operator's fingers. When fingers draw close and the scissor would be closed too, as shown in Figure 10. And Figure 11 shows the simulation of extraocular muscle cutting. 


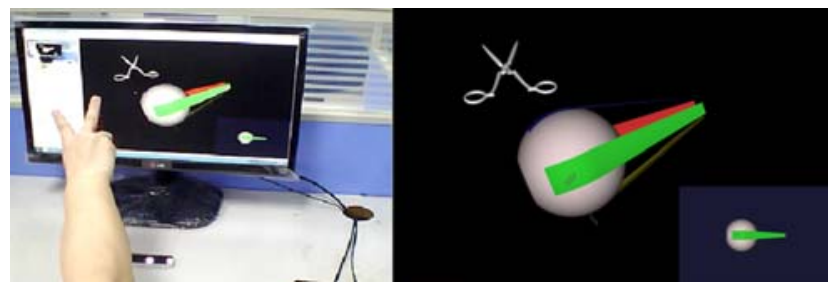

FIGURE IX. THE RIGHT HAND GESTURE SHEAR OPEN

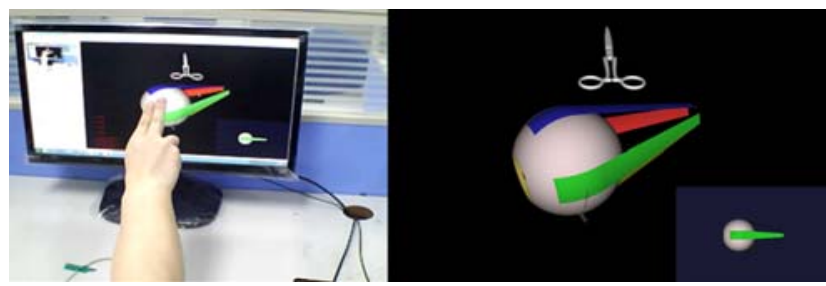

FIGURE X. THE RIGHT HAND GESTURE SHEAR CLOSED

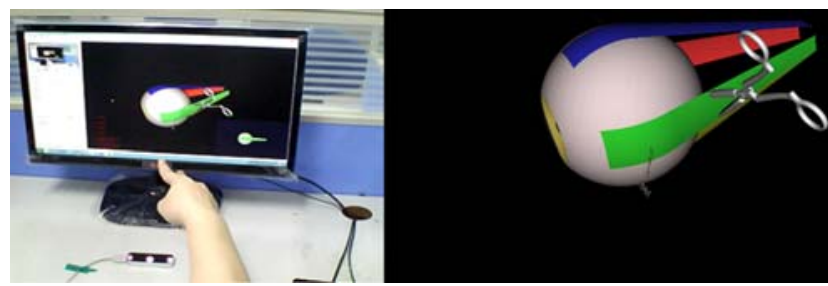

FIGURE XI. THE SIMULATION OF CUTTING OPERATION

\section{The Analyses of Kalman Filter}

When we move the virtual scalpel by the right hand gesture Direct, the position data of scalpel are optimized by Kalman Filter. And the measuring path of the scalpel in the process of virtual knifing is showed in the Figure 12. After using Kalman Filter to optimize the measuring path, the knifing path is showed in the Figure 13. Compared with the two kinds of knifing paths, we can know that the knifing path is more smooth and stable.

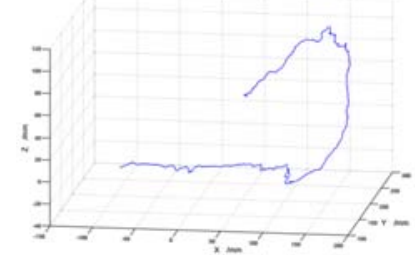

(A)

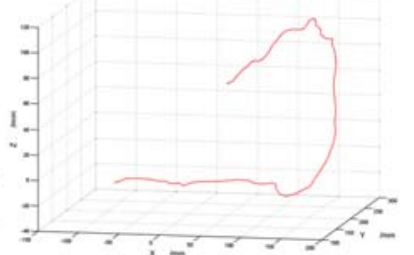

(B)
FIGURE XII. (A )THE MEASURING PATH OF THE SCALPEL IN THE PROCESS OF VIRTUAL KNIFING: (A) ORIGINAL (B) OPTIMIZED BY KALMAN FILTER

\section{DISCUSSIONS}

The collision detection is the key part of the virtual surgery. In the experiments, we use the OBB bounding box to simulate the collision detection in the surgery. But the OBB bounding box is not suitable for the collision detection in the shape change process of soft tissue. In order to improve the reality of virtual surgery, a new and high effect method of collision detection should be considerate.

The paper uses the gesture to realize the human-robot interaction in the process of virtual surgery. And the user can enjoy a great interaction experience and vision feeling. However, the touch and auditory sense experience were not provided in the system. These will be added to the system in the future work.

\section{CONCLUSION}

The paper presents a key technology research of virtual surgery based on gesture interaction. And this paper makes a conclusion to the usual gesture and builds the gesture library which can be applied to the system of virtual surgery. Leap Motion is used to collect the gesture data. The paper maps the five kinds of gesture in the gesture library into the concert control operation of the virtual surgery. Besides, different hands were defined different operations. Therefore, the doctor can avoid the touch with the virtual surgery instrument. We provide a simpler and more natural operation method of virtual surgery. Kalman Filter and Unscented Kalman Filter were used in the paper to process the original gesture data collected by Leap Motion. It makes the movement of surgery instrument more accuracy and stable in the process of surgery. Finally, experiments were performed to show the effectiveness of the system. And experimental results demonstrated that this system could be applied to the real situation of surgery.

\section{ACKNOWLEDGMENT}

Project funded by "Guangdong Provincial Science and Technology Project (2014B090921007)" and "Guangzhou Municipal Science and Technology Program (20150810068)"

\section{REFERENCES}

[1] Medellin-Castillo, H.I., et al. "The evaluation of a novel haptic-enabled virtual reality approach for computer-aided cephalometry,” Computer Methods \& Programs in Biomedicine, vol.130, pp.46-53, 2016.

[2] Mylavarapu, G, et al. "Computational Modeling of Airway Obstruction in Sleep Apnea in Down Syndrome: A Feasibility Study," Otolaryngology Head \& Neck Surgery, vol. 155, pp. 184-187, 2016.

[3] R. M. Satava and S. B. Jones, "Current and future applications of virtual reality for medicine,” Proceedings of the IEEE, vol. 86, no. 3, pp. 484489, 1998.

[4] Touch surgery simulator. [Online]. Available: https://www.touchsurgery.com/

[5] Virtual reality surgery with gopro and oculus rift. [Online]. Available: http://www.htxt.co.za/2014/08/18/virtual-reality-surgery-with-goproandoculus-rift/

[6] C. Graetzel, T. Fong, S. Grange, and C. Baur, "A non-contact mouse for surgeon-computer interaction,” Technology and Health Care, vol. 12,no. 3, pp. 245-257, 2004.

[7] G. Marin, F. Dominio, and P. Zanuttigh, "Hand gesture recognition with jointly calibrated leap motion and depth sensor," Multimedia Tools and Applications, pp. 1-25, 2015.

[8] D. Bassily, C. Georgoulas, J. Guettler, T. Linner, and T. Bock, "Intuitive and adaptive robotic arm manipulation using the leap motion controller,"in ISR/Robotik 2014; 41st International Symposium on Robotics; Proceedings of. VDE, 2014, pp. 1-7.

[9] E. Artemciukas and L. Sakalauskas, "Leap motion controller application in augmented reality technology,” Technologijos mokslo darbai Vakaru Lietuvoje, vol. 9, pp. 10-15, 2014. 
[10] G. Welch and G. Bishop, "An introduction to the kalman filter. department of computer science, university of north carolina," 2006.

[11] T. Schmidt, F. P. Araujo, G. L. Pappa, and E. R. Nascimento, "Real-time hand gesture recognition based on sparse positional data,” Technology \& Health Care Official Journal of the European Society for Engineering \& Medicine, vol. 12, no. 3, 2004.

[12] R. Lun and W. Zhao, “A survey of applications and human motion recognition with microsoft kinect," International Journal of Pattern Recognition and Artificial Intelligence, vol. 29, no. 05, p. 1555008, 2015.

[13] M. Sathiyanarayanan and T. Mulling, "Map navigation using hand gesture recognition: A case study using myo connector on apple maps,”Procedia Computer Science, vol. 58, pp. 50-57, 2015.

[14] S. Iba, J. M. V. Weghe, C. J. Paredis, and P. K. Khosla, “An architecture for gesture-based control of mobile robots," in Intelligent Robots and Systems, 1999. IROS'99. Proceedings. 1999 IEEE/RSJ International Conference on, vol. 2. IEEE, 1999, pp. 851-857.

[15] G. Du and P. Zhang, "Markerless human-robot interface for dual robot manipulators using kinect sensor," Robotics and Computer-Integrated Manufacturing, vol. 30, no. 2, pp. 150-159, 2014. 